\title{
Réplicas en Redes Sociales que Denuncian Desigualdades de la Estrategia \#AprenderDigital en Tiempos de Covid-19
}

\author{
Social Network Replies that Denounce Inequalities of the \\ \#AprenderDigital Strategy in Time of Covid-19
}

\author{
Edwar Camilo Lopera-Zuluaga * \\ Universidad Pontificia Bolivariana, Colombia
}

\begin{abstract}
El Ministerio de Educación colombiano publicita en redes sociales la estrategia \#AprenderDigital para contrarrestar el cierre masivo de escuelas por el covid-19. No obstante, la ciudadanía hace réplica inconforme a esta homogénea iniciativa. Esta investigación de enfoque cualitativo pretende, primero, clasificar en categorías de análisis las réplicas ciudadanas realizadas a las publicaciones del Ministerio Nacional de Colombia en Twitter y Facebook bajo la etiqueta \#AprenderDigital. Segundo, discutir, en el marco de las réplicas ciudadanas, las expresiones de desigualdad educativa exacerbadas por la estrategia digital, además, de ofrecer algunas lecciones aprendidas para la política educativa digital pospandemia en Colombia. El método implicó recuperar las réplicas ciudadanas y los datos con apoyo del software de análisis cualitativo Atlas ti. Las réplicas fueron clasificadas en tres categorías: 1. Réplicas de acceso. 2. Réplicas curriculares. 3. Exhortaciones. Hacia el final, discutimos sobre la fortaleza del estudio para ratificar la desigualdad educativa en tiempos de covid-19, la homogeneidad de las políticas educativas de emergencia que niegan las diferencias, reflexionamos sobre las necesarias políticas de subjetividad y aportamos algunas reflexiones sobre la dirección de las políticas digitales pospandemia. Por último, valoramos la fertilidad que significa recuperar réplicas en redes sociales para la investigación en ciencias sociales.
\end{abstract}

Descriptores: Brecha digital; Covid-19; Estrategia educativa; Red social; Réplicas ciudadanas.

The Colombian Ministry of Education publishes the \#AprenderDigital strategy on social networks to counteract the massive closure of schools due to covid-19. However, the public makes a dissatisfied reply to this homogeneous initiative. This research with a qualitative approach aims, first, to classify in categories of analysis the citizen replies made to the publications of the National Ministry of Colombia on Twitter and Facebook under the hashtag \#AprenderDigital. Second, to discuss, within the framework of citizen replies, the expressions of educational inequality exacerbated by the digital strategy, in addition to offering some lessons learned for post-pandemic digital educational policy in Colombia. The method involved retrieving the citizen replicas and the data with the support of the qualitative analysis software Atlas ti. The replicas were classified into three categories: 1. Access replicas. 2. Curricular replicas. 3. Exhortations. To the end, we discuss the strength of the study to ratify educational inequality in times of covid-19, the homogeneity of emergency educational policies that deny differences, we reflect on the necessary subjectivity policies and we provide some reflections on the direction of postpandemic digital policies. Finally, we value the fertility that means recovering replicas in social networks for research in social sciences.

Keywords: Digital gap; Covid-19; Educational strategy; Social network; Citizen replies.

*Contacto: edwar.lopera@upb.edu.co

ISSN: 2254-3139

www.rinace.net/riejs/

revistas.uam.es/riejs
Recibido: 15 de mayo 2020

$1^{\text {a }}$ Evaluación: 13 de agosto 2020

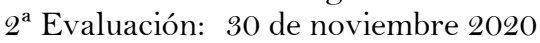

Aceptado: 15 de diciembre 2020 


\section{Introducción}

El 15 de marzo de 2020 en alocución presidencial, el presidente de la República y la Ministra de educación de Colombia confirmaron lo que parecía inminente: el cierre masivo de las escuelas primarias y secundarias para confrontar la expansión de la pandemia covid19. La estrategia anunciada para garantizar la continuidad de las clases a cerca de 10 millones de niños, niñas y jóvenes, además para apoyar el trabajo docente fue denominada \#AprenderDigital. La estrategia, de acuerdo con el comunicado de prensa del 16 de marzo de 2020 del Ministerio, es una

(...) plataforma de contenidos educativos digitales de todas las áreas del conocimiento
para estudiantes de los grados escolares. Los usuarios pueden acceder y sin necesidad
de registro, podrán encontrar adicionalmente una selección de plataformas,
aplicaciones, videojuegos educativos, y cursos virtuales abiertos, de manera gratuita
para todos (...) En este espacio los docentes de cualquier nivel educativo: preescolar,
básica primaria, secundaria o media encontrarán contenidos educativos interactivos
y de fácil uso para trabajar con sus estudiantes. Podrá enviarlos por correo electrónico,
integrarlos a la plataforma educativa del colegio, en algunos casos descargarlos para
trabajarlos de manera offline. (Ministerio de Educación de Colombia, 2020, párr.
1)

La estrategia fue acompañada de un eslogan que rezaba "contenidos para todos". No obstante, la estrategia, mucho menos el eslogan, tuvieron fácil aceptación entre la ciudadanía que denunciaba cierta homogeneidad y solucionismo tecnológico para enfrentar el cierre de las escuelas, mientras las cifras del Departamento Administrativo Nacional de Estadística (2018) expresan que, en el total nacional, 44,9\% de las personas de 5 y más años usaron computador y 64,1\% usaron Internet en cualquier lugar. Esta inconformidad ciudadana tuvo resonancia en las redes sociales oficiales de Twitter y Facebook del Ministerio, a través de réplicas a las constantes publicaciones construidas con sarcasmos y memes de alto valor empírico.
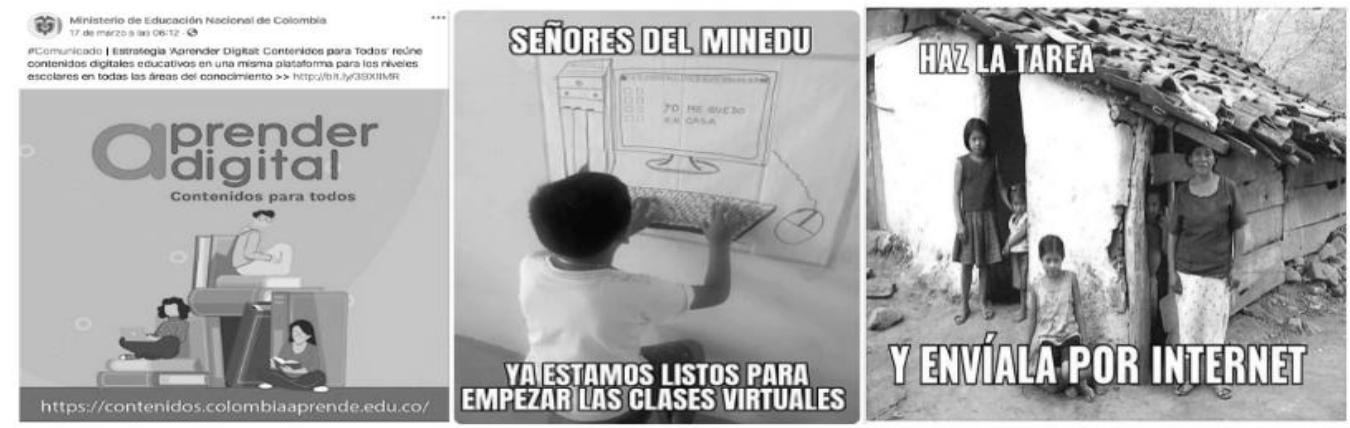

Figura 1. Primera imagen corresponde a publicación del 17 de marzo de 2020 del Ministerio de Educación. Segunda y tercera corresponden a réplicas ciudadanas a la publicación (de izquierda a derecha)

Fuente: Twitter@Mineducación marzo 17 de 2020.

En consecuencia, el presente ejercicio de investigación tiene dos objetivos: primero, categorizar las réplicas ciudadanas realizadas a las publicaciones oficiales del Ministerio Nacional de Colombia en Twitter y Facebook bajo la etiqueta \#AprenderDigital. Segundo, discutir, en el marco de las réplicas, las expresiones de desigualdad educativa reveladas por la estrategia digital, además, de ofrecer algunas lecciones aprendidas para la política educativa digital pospandemia en Colombia. Para estos fines, en la primera parte 
revisamos literatura en torno a la relación educación y covid-19 para Latinoamérica. En la segunda parte, presentamos el método de investigación destacado por su carácter cualitativo y afinado de cara a la imposibilidad del contacto físico para la recolección de información. En la tercera parte, presentamos resultados matizando la interpretación del investigador y el candor de algunas réplicas ciudadanas ejemplificantes que fundamentan los argumentos. Por último, discutimos los resultados en el marco de la literatura revisada y concluimos con algunas fortalezas y lecciones de la presente investigación.

\section{Revisión de literatura}

Si de algo podemos tener certeza en tiempos de excepcionalidad, es que las políticas educativas digitales latinoamericanas tuvieron una prueba de fuego sin parangón. Unas bajo el énfasis de la tecnología educativa tradicional, otras apegadas a los programas 1 a 1, y finalmente, otras con aspiraciones a la formación del pensamiento computacional (Lugo et al., 2020). Sin lugar a error, podemos anticipar que los próximos años será trabajo de los sistemas educativos nacionales corregir las brechas exacerbadas que dejó el paso de la pandemia y autocriticar si los énfasis de política digital otorgados hasta entonces fueron suficientes, además de pertinentes, para salvaguardar el derecho a la educación de niñas y niños.

Hay consenso en la literatura sobre las desigualdades educativas acentuadas en época covid-19. Sánchez-Gómez y colaboradores (2020) evidencia cómo la cuarentena significó mayor dificultad para el acceso a todos los servicios educativos. Nada desconocido, aunque paradójico para el caso de la política digital, tan connotada, publicitada y aceptada casi por unanimidad, que demostrara tan graves limitaciones para atender la contigencia. En particular, el reguero de pólvora (Rivas, 2015), que significó la moda digital en América Latina, quedó por estos días explícito que no era a tal magnitud. La inversión digital tuvo restricciones territoriales para los sectores sociales menos favorecidos que ven hoy por hoy agravada la brecha de aprendizajes, la facilidad para el acceso a la información y limitadas posibilidades de familias con el suficiente capital tecnológico para acompañar los procesos educativos (Garrido, 2020; Quiroz, 2020).

El fenómeno de segregación escolar en América Latina, los últimos años de creciente diagnóstico en la literatura (Krüger, 2019; Murillo y Martínez-Garrido, 2017, 2018), encuentra mayor intensidad por estas fechas. Los resultados escolares, una de las evidentes consecuencias del fenómeno segregador, se agravan porque están condicionados al acceso, o no, a los recursos digitales para paliar el cierre de las escuelas (Álvarez et al., 2020). Una vez más, la segmentación público-privada del sistema educativo deja en evidencia para el sector público los peores réditos por agrupar la población más pobre y deja, una vez más, bien publicitada a la educación privada como solución a los problemas de la educación.

Una constante revisada en la literatura es la homogeneidad y el solucionismo tecnológico característicos de los programas educativos para contrarrestar el cierre masivo de las escuelas. El estudio de Cáceres-Muñoz, Jiménez-Hernández y Martín-Sánchez (2020) evidencia el giro intempestivo de los programas educativos hacia una inusitada transformación digital de la escuela. Nada extraño por estas épocas insistir en la escuela digital, sin embargo, la unívoca respuesta digital a la contigencia significa dejar a muchos sin voz, impávidos, sin capacidad de respuesta. Entonces, resulta peligroso adjudicar per se a la tecnología una garantía de solución al problema actual de la educación (Álvarez et 
al., 2020). Queda el sabor de boca de programas educativos de emergencia a la medida de una homogénea masa de población caracterizada por acceder a los recursos digitales, pero alejados de otra que, heterogénea y en contextos sociales diversos, ve las brechas educativas exacerbadas (Mérida y Acuña, 2020).

En el estudio de Gallegos y Tinajero (2020) encontramos cómo la homogeneización de los programas educativos para enfrentar la contigencia refleja a contraluz siluetas que no se ajustan a la medida de las pretensiones. La difuminada contraluz devela altos niveles de analfabetismo y limitantes de acceso digital, además de desfase entre los diseños de las estrategias educativas y los contextos de actuación de los ciudadanos de a pie inclinados antes que, a la prioridad educativa, hacia la incertidumbre por la salud y el empleo en vilo. A esta imagen a contraluz, Garrido (2020) agrega la necesidad de que las escasas medidas educativas con intención de subsanar las desigualdades escalen más allá del período de contigencia y no obedezcan a voluntades transitorias, sino que derive en un viraje hacia el apoyo permanente a los excluidos. Es el mismo llamado revisado en Rodríguez (2020) hacia políticas educativas focalizadas y ampliadas para afrontar las brechas actuales y las que, en perspectiva, significarán la pospandemia. Significa entonces, no dejar atrás a los más débiles a partir de educar una mirada desigual: aquella para no tratar por igual a los desiguales sino para ofrecer más a quien más lo solicita (Cáceres-Muñoz, JiménezHernández y Martín-Sánchez, 2020).

Terminemos por revisar el lugar de la institución escolar en esta situación de excepcionalidad educativa. La literatura coincide en la misión de contención social de la escuela capaz de apoyar la limitada posibilidad de acompañamiento escolar en muchas familias o de compensar de forma creativa la inaccesibilidad digital para continuar las clases. En particular, hay consenso en la figura del profesor, por estos días restituida, porque está en la primera línea de contacto con los más desfavorecidos, impulsa a no fallar y a paliar la situación de indefensión de muchos (Cáceres-Muñoz, Jiménez-Hernández y Martín-Sánchez, 2020; Cervantes y Gutiérrez, 2020). El profesor es actor clave que ejerce su labor al ritmo de sus propias angustias existenciales: problemas logísticos, tecnológicos y socio afectivos. Procura equilibrios expresados en la priorización curricular versus las necesidades propias de su contexto escolar, o la impotencia para ofrecer la debida retroalimentación del aprendizaje a sus estudiantes (Ramos-Huenteo et al., 2020; Sánchez et al., 2020). La labor contentiva de la escuela hoy por hoy no es una abstracción institucional sino materialidad hecha docencia capaz de imaginar la práctica en tiempos adversos, recrear redes de comunicación para rectificar la forzosa ruptura escuelacomunidad. Para caminar entre la resistencia a no dejar el tradicional arte de la docencia aprendida y los nuevos retos que significa trabajar a la distancia (Baptista et al., 2020).

Nos preguntamos, una vez revisada la situación investigativa sobre educación y covid-19 en latinoamericana, ¿cuál es la situación particular de la estrategia \#AprenderDigital, programa educativo dirigido a contrarrestar el cierre masivo de las escuelas para más de 10 millones de niñas y niños en Colombia? Por tanto, nos proponemos una investigación exploratoria y cualitativa para, primero, categorizar las réplicas ciudadanas realizadas a las publicaciones oficiales del Ministerio Nacional de Colombia en Twitter y Facebook bajo la etiqueta \#AprenderDigital. Segundo, discutir, en el marco de las réplicas, las expresiones de desigualdad educativa reveladas por la estrategia digital, además, de ofrecer algunas lecciones aprendidas para la política educativa digital pospandemia en Colombia. 


\section{Método}

\section{Enfoque metodológico}

El estudio es cualitativo. Taylor y Bodgan (1987) valoran de este enfoque la obtención de significados a través del ejercicio sensible de ir hacia la gente. Es paradójico, la motivación del presente ejercicio resultó de la imposibilidad de establecer contacto físico con los otros porque la disposición actual al distanciamiento social propone un estilo de vida confinado y aislado para preservar la vida propia y la ajena. Entonces, bajo estas condiciones, surgió el interrogante sobre ¿cómo obtener significados acerca del fenómeno masivo de cerrar escuelas? Este problema obligó abrir perspectivas para no dejar de escuchar la gente y afinó el método. Entonces, el espacio a las réplicas en las redes de sociales de Twitter y Facebook resultó ser de un alto valor empírico para auscultar las denuncias, y con éstas, aportar al ejercicio de advertir las implicaciones que hoy por hoy tiene cerrar las escuelas a más de 1.000 millones de niños, niñas y jóvenes en el mundo entero (UNESCO, 2020a).

\section{Procedimiento}

Recuperamos 169 réplicas ciudadanas a las publicaciones del Ministerio de Educación Nacional bajo la etiqueta \#AprenderDigital, rastreadas en las redes sociales de Twitter y Facebook entre el 15 de marzo y el 30 de abril. Lapso que coincidió con la promoción de la estrategia gubernamental como respuesta al cierre masivo de las escuelas colombianas.

Cuadro 1. Indicador de réplicas clasificadas para cada categoría y fechas de recuperación

\begin{tabular}{lccccc}
\hline \multirow{2}{*}{ CATEGORÍA } & $\begin{array}{c}\# \mathbf{1 5 - 2 1} \\
\text { MARZO }\end{array}$ & $\begin{array}{c}\text { \#22-28 } \\
\text { MARZO }\end{array}$ & $\begin{array}{c}\text { \#29MARZO-4 } \\
\text { ABRIL }\end{array}$ & $\begin{array}{c}\text { \#5-30 } \\
\text { ABRIL }\end{array}$ & TOTALES \\
\hline Acceso & 15 & 13 & 7 & 14 & $49(28.9)$ \\
\hline Currículo & 18 & 10 & 15 & 16 & $59(34.9)$ \\
\hline Exhortaciones & 21 & 21 & 10 & 9 & $61(36)$ \\
\hline Totales & $54(31,9)$ & $44(26)$ & $32(18.9)$ & $39(23)$ & $169(100)$ \\
\hline
\end{tabular}

Fuente: Elaboración propia.

\section{Gestión de los datos}

Gestionamos los datos con el software de análisis cualitativo Atlas ti 8. Las réplicas de Twitter fueron recuperadas a través de la función automática Importar desde Twitter. Por su parte, los datos recuperados de Facebook fueron incluidos al software de manera manual en un documento primario de procesador de texto. Agrupamos las réplicas de Twitter en un solo documento primario 1 y las réplicas de Facebook en un documento primario 2. Para facilitar la identificación y presentación de los resultados etiquetamos las réplicas bajo el código atributivo \#fecha. Por ejemplo, léase la cita 1:8, \#15marzo, así:

- 1 para documento primario 1 que corresponde a réplicas recuperadas de Twitter

- 8 para ubicación de la réplica dentro del documento primario 1

- \#15marzo para fecha de publicación de la réplica

\section{Análisis de los datos}

Asumimos la propuesta de análisis cualitativo NCT -Noticing, Collect, Thinking- (Friese, 2019). Primero, notamos cosas interesantes a través de la lectura y codificación inicial (Charmaz, 2006), de las 169 réplicas ciudadanas recuperadas de Twitter y Facebook. Esta primera etapa arrojó 38 códigos que resumieron temáticamente el corpus documental y nos proporcionaron una visión general la información. Sin embargo, nos encontramos con 
el denominado Code Swamp o pantano de códigos caracterizado por una alta descripción o cercanía a los datos (Friese, 2019).

Cuadro 2. Resultado de codificación inicial o pantano de códigos descriptivos

\begin{tabular}{l}
\hline CóDIGos \\
\hline Acceso: desde la periferia indígena; Acceso: desde la pobreza; Acceso: desde la ruralidad; \\
Acompañamiento del hogar; Asegurar necesidades básicas; Buscar equidad; Compromiso \\
intersectorial; Currículo: acompañar desde el hogar; Currículo: capacitar y virtualizar \\
costumbres; Currículo: desarrollar habilidades desde casa; Currículo: diseñar un modelo \\
alternativo de educación; Currículo: inclusión; Currículo: privilegiar unos saberes; Currículo: \\
subutilizar el recurso vigente; Currículo: depender matriz tradicional; Currículo: pertinencia; \\
Denuncias curriculares; Digitalizar costumbres; Diseñar modelos alternativos; Disposiciones; \\
Estrategia distorsionada; Exhortaciones; Expandir fronteras escolares; Inclusión; La situación \\
precaria del maestro periférico; Mayor inversión del estado; País visibilizado y país \\
invisibilizado; Planeación con enfoque territorial; Política digital restringida; Privilegiar \\
saberes; Reconocimiento internacional; Sugerencias: aumentar inversión del estado; \\
Sugerencias: buscar equidad; Sugerencias: cerrar la brecha digital; Sugerencias: compromiso \\
intersectorial; Sugerencias: pensar en los contextos sociales; Sugerencias: privilegiar necesidades \\
básicas.
\end{tabular}

Fuente: Elaboración propia.

Segundo, recolectamos códigos que albergaban significados similares o podían agruparse en códigos con mayor nivel de abstracción. Iteramos sobre la literatura revisada y clasificamos los códigos bajo las categorías de acceso y currículo. Los códigos agrupados en acceso reunían los malestares de las promesas incumplidas de universalidad digital en América Latina, la homogenización de las políticas digitales o la carencia de mirada territorial para el acceso. De su parte, los códigos agrupados en currículo expresan el reto de la transformación digital de la escuela, el rol de los actores educativos; en especial, la familia y el maestro y la necesaria ampliación de la noción de escuela con apertura al ecosistema digital actual. Para este movimiento analítico hicimos vigilancia metodológica a la construcción categorial a través del principio de mutua exclusividad expuesto por Freeman (2017).

Cuadro 3. Descripción de las categorías construidas a partir de las réplicas ciudadanas

\begin{tabular}{ll}
\hline \multicolumn{1}{c}{ CATEGORÍA } & \multicolumn{1}{c}{ CONTENIDOS } \\
\hline \multirow{3}{*}{ Réplicas de acceso } & Estrategia distorsionada \\
& País visibilizado y país invisibilizado \\
& Política digital restringida \\
& La situación precaria del maestro periférico \\
\hline & Digitalizar costumbres \\
& Inclusión \\
& Acompañamiento del hogar \\
& Privilegiar saberes \\
Réplicas curriculares & Expandir fronteras escolares \\
& Diseñar modelos alternativos \\
\hline & Buscar equidad \\
& Planeación con enfoque territorial \\
& Mayor inversión del Estado \\
Exhortaciones & Asegurar necesidades básicas \\
& Compromiso intersectorial \\
\hline
\end{tabular}

Fuente: Elaboración propia.

En consecuencia, la información no contenida bajo ninguna de estas dos categorías implicó construir una tercera denominada Exhortaciones que recogía tonalidades y matices 
ciudadanos dirigidos a la sugerencia, la propuesta futura y la recomendación para que el Estado vire hacia las necesidades más sentidas de la población.

Finalmente, pensamos en torno a las réplicas contenidas en cada categoría. Por tanto, apelamos a la escritura de memos analíticos para describir, interpretar y relacionar el contenido de las categorías a la luz de la literatura revisada. Adicional, empleamos la visualización de datos en redes semánticas para fundamentar los argumentos basados en réplicas ejemplares (Charmaz, 2006).

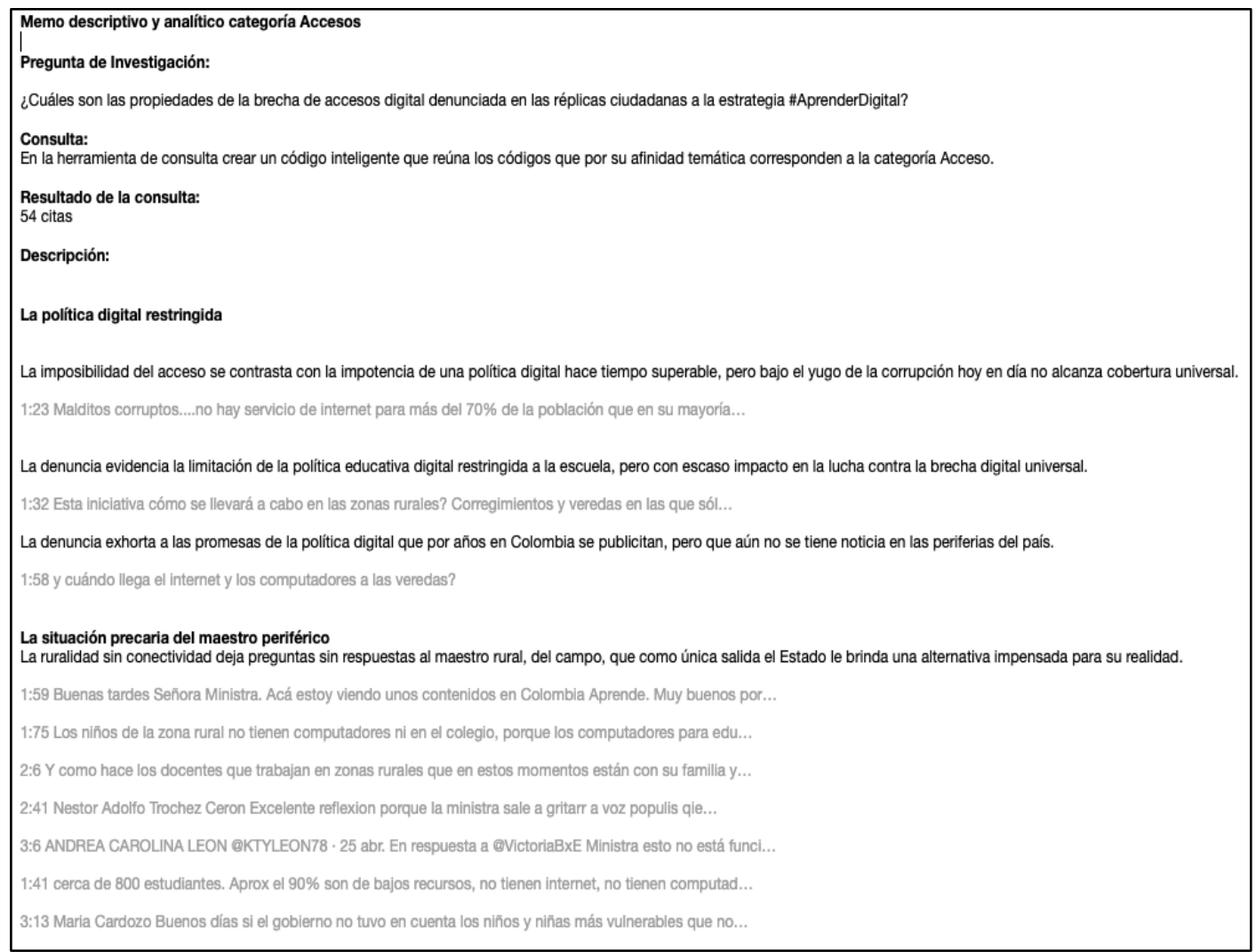

Figura 2. Fragmento de un memo analítico

Fuente: Elaboración propia.

\section{Resultados}

En el presente acápite devalamos el contenido las tres categorías construidas a partir de las réplicas ciudadanas recuperadas en Twitter y Facebook. Escribimos matizando entre la interpretación del investigador y al candor de las réplicas ciudadanas.

\subsection{Réplicas de acceso}

\subsubsection{Estrategia distorsionada}

La estrategia digital se considera buena e innovadora, pero se distorsiona porque todos y todas no tienen acceso a Internet desde el hogar. La estrategia parece pensada para una tipología de hogar: el urbano, pero se aleja de los hogares que habitan veredas y corregimientos lejanos a las urbes. La ruralidad reclama la pertinencia social de las estrategias educativas pensadas desde la lejanía y la comodidad burocrática, pero con escasa sensibilidad por el contexto particular. 
En las zonas rurales no tiene los equipos no cuentan con el servicio de internet muy claro que están sentados en sus buenas oficinas y en la ciudad se les olvida que existe el campo. (1:81, \#25marzo)

La estrategia desconoce la condición de familias sin la capacidad para acompañar y desarrollar aprendizajes virtuales, además parece dar la espalda a las prioridades del proyecto de vida rural. Además, depende de conectividad, pero no contempla accesos sin uso de datos de Internet. Las familias sin recursos económicos no logran costear la conectividad.

En el caso de las áreas rurales no hay conectividad en su gran mayoría. Entonces los niños se encuentran aislados tecnológicamente. Si un padre de familia del área rural tiene para el sustento de su familia no va a tener para brindarle ayudas tecnológicas a sus hijos (2:8, \#6abril).

\subsubsection{Política digital restringida}

La imposibilidad del acceso se contrasta con la impotencia de una política digital restringida que hace tiempo pudo ser alcanzable, pero bajo el yugo de la corrupción hoy en día aún no alcanza cobertura universal. La denuncia evidencia la limitación de la política educativa digital restringida a la escuela, pero con escaso impacto en la lucha contra la brecha digital universal. La denuncia exhorta a las promesas de la política digital que por años en Colombia se publicitan, pero que aún no se tiene noticia en las periferias del país.

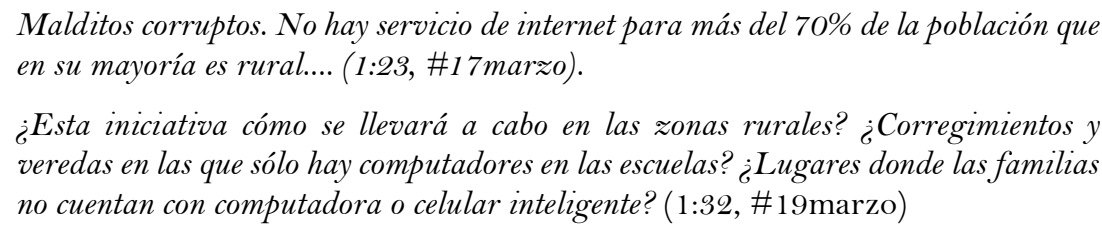

4.1.3. País visibilizado y país invisibilizado

El reclamo de la conectividad campea de norte a sur y denuncia la cara invisible de la periferia colombiana. Al sur, Amazonas poco aparece en las decisiones, ni en las políticas de un país centralista y urbano. El Amazonas donde no están garantizados los servicios básicos tiene que ver con resignación la incompatibilidad de la propuesta digital. Al norte, la noticia del acceso digital como solución al cierre de las escuelas para las comunidades indígenas Wayuú, se confronta con el escaso acceso al agua y la seguridad alimentaria de niños y niñas.

En las comunidades indígenas del Amazonas no cuentan con energía las 24 horas. Siendo Leticia la capital la conectividad es pésima. Las familias del Amazonas no todos cuentan equipos de computación. Ahi dejo la inquietud y espero una amable respuesta y posibles soluciones. Colombia. (1:76, \#24marzo)

$\mathrm{El}$ éxito de la estrategia \#AprenderDigital para atender el cierre masivo de escuelas se publicita y es reconocido en los medios de comunicación internacional, pero la pregunta del ciudadano increpa: ¿a qué tipo de país estará haciendo alusión la estrategia? La denuncia contempla la distancia entre el desfavorecido y el privilegiado encarnados en la dicotomía centro-periferia. La estrategia desconoce al campesino que nutre la despensa del país, pero al que más tarde le llegan las oportunidades digitales. La pandemia es la oportunidad para que los dirigentes recuerden el otro país para el cual la solución tecnológica no es la salida unívoca al cierre de las escuelas. 
La@bbcmundo resalta hoy la labor que adelanta@Mineducacion a través de \#AprenderDigital, la plataforma para que estudiantes, docentes y padres de familia accedan a contenidos educativos. Un reconocimiento a la educación virtual en medio de la crisis por el Covid-19. (3:4, \#24abril)

¿Qué estrategias nos brinda el ministerio de educación para la población de estudiantes que viven en zonas rurales donde no hay conectividad ni señal telefónica? Es fácil pensar en la población urbana, pero se discrimina a la población rural. (1:86, \#26marzo)

\title{
4.1.4. La precaria situación del maestro periférico
}

La ruralidad sin conectividad deja preguntas sin respuestas al maestro rural al cual el Estado le brinda una alternativa impensada para su realidad.

\begin{abstract}
Buenas tardes, Señora Ministra. Acá estoy viendo unos contenidos en Colombia Aprende. Muy buenos, por cierto. Pero como hago para que mis estudiantes de la básica secundaria accedan a ellos cuando ni siquiera la institución cuenta con conectividad mucho menos los estudiantes campesinos. Ni siquiera todos cuentan con dispositivos móviles. Le hecho cabeza y no encuentro solución aún. (1:59, \#21 de marzo)
\end{abstract}

\subsection{Réplicas curriculares}

\subsubsection{Acompañamiento del hogar}

La tecnología digital reta a las familias a un escenario incierto: pasar del acompañamiento familiar en una escuela conocida y calculada por todos, a una versión de escuela que puede estar en casa y requiere conocimientos de las familias para poder acompañar. El rol de profesor se difumina: ¿qué rol desempeña el profesor desde la distancia cuando la presencia no está garantizada? El rol del profesor parece resistirse en las formas tradicionales y entra en confrontación con las nuevas disposiciones de la no presencialidad. La representación familiar del profesor aún es de dependencia y no la del actor para el trabajo colaborativo con ésta.

Culturalmente las comunidades dependen de la explicación del profesor impidiendo el desarrollo de la metodología de aprendizaje autónomo y colaborativo. (1:84, \#25marzo)

\subsubsection{Expandir fronteras escolares y digitalizar costumbres}

La estrategia digital interpela la escuela tradicional que parece señalada; está en evidencia porque no hay murallas que definan el adentro y el afuera de la escuela. Las fronteras del conocimiento se expanden más allá del edificio escolar. El docente es interpelado a cambiar de chip, lo tiempos no son los mismos. El consuelo del maestro interpelado es reconocer que, en estas circunstancias excepcionales de la pandemia, todos los oficios están nominados a la transformación, la flexibilización, la capacitación y a (re)crear costumbres afines con las tendencias futuras.

Una vez más invitándoles a que aprovechen este recurso para cerrar la brecha digital de docentes del municipio y se pongan en la tarea de cambiar el chip, inclusive es útil para todos los que sean padres y expandan las fronteras del conocimiento. (1:22, \#17marzo)

Las transformaciones requieren no solo voluntades individuales sino políticas que valoren la inversión para transformar ambientes de aprendizaje que dispongan nuevas formas de interactuar, nuevos roles y flujos a través de los cuales circule la información y el conocimiento. Con esto, la capacitación docente para lograr apertura a cambios y nuevas formas de orientar la labor educativa. 
En estos momentos se evidencia la necesidad de invertir en educación, con ambientes de aprendizaje que incluyan herramientas tecnológicas que permitan la interacción entre docentes, estudiantes y padres de familia en todas las instituciones educativas del país. Además de la capacitación que se debe brindar a los docentes en el uso de plataformas virtuales acorde a las necesidades de su contexto escolar. (1:60, \#21marzo)

\subsubsection{Diseñar modelos alternativos}

Un modelo alternativo para la educación de los niños y las niñas se ve venir a través de la integración de todos los medios de comunicación tradicionales y digitales como una plataforma educativa nacional que contribuya a la labor de la escuela. El rol de la universidad debe estar en adelante más comprometido con el acompañamiento a las escuelas en dirección a proponer modelos alternativos de escuela, porque el modelo no se agota en la provisión de recursos, sino con la articulación de voluntades. La universidad debe articularse a la toda la escuela, es decir, no limitarse a los ciclos previos al primer año de la universidad.

Como ciudadanos deberíamos proponer como parte del aprendizaje de este momento histórico, que estos programas educativos para niños sean una constante, una alternativa para los niños en la programación de la tv Nacional. ;Mucho mejor que programas de chisme, nada productivos o novelas que enseñan la mayoría modelos inadecuados o niños que a corta edad están ansiosos por conectarse al celular y expuestos a la pornografia que hasta los adultos han normalizado ignorando las graves secuelas! Que tal si los miramos y proponemos continuidad como ganancia en tiempos dificiles. (1:48, \#18marzo)

Poseer la conectividad y asumir el reto la educación desde la casa da lugar a comprender que la escuela puede tener varios eslabones, varias estaciones de un viaje a gran distancia por el cual muchos actores contribuyen y acompañan la formación. El Covid-19 es la oportunidad de las familias para comprender que también pueden aprender con los niños y las niñas. Qué la educación está restringida a la escolarización con sus rutinas disciplinares, ciclos y organización, ni mucho menos termina con el ritual de paso que significa la certificación. Qué la educación puede ser una plataforma de integración entre escuela y familia.

Una vez más invitándoles a que aprovechen este recurso para cerrar la brecha digital de docentes del municipio y se pongan en la tarea de cambiar el chip, inclusive es útil para todos los que sean padres y expandan las fronteras del conocimiento. (1:22, \#17marzo)

4.2.4 Privilegiar saberes

La propuesta digital sigue compartimentando el currículo en disciplinas. Con ello, jerarquizando asignaturas en nivel de importancia. A pesar de los tiempos difíciles que requieren otras reflexiones, parecen ser más necesarios los saberes útiles, los evaluados en pruebas. La preocupación parece estar más dirigida a no perder el tiempo y garantizar las disciplinas de verdad importantes, que en fomentar saberes necesarios para los tiempos que estamos viviendo.

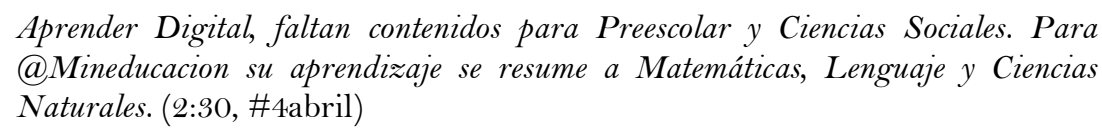

\subsubsection{Inclusión}

Las personas en situación de discapacidad reclaman espacio en la estrategia digital. En la escuela tradicional su estancia es hostil porque no acceden a los niveles de "normalidad" 
que espera la escolarización. En la estrategia digital se perciben exacerbadas las brechas que apenas la escuela presencial intenta cerrar. El exceso de un formato visual es confrontado por la discapacidad visual. ¿Cómo propiciar una estrategia digital razonablemente adaptada a las necesidades de la discapacidad en todas sus manifestaciones? ¿Cómo promover la estrategia digital como posibilidad de una escuela más justa con las diferencias? Pareciera que se cambia de formato, pero se continúan acentuando las mismas desigualdades y perfilando una sola manera de ser apto para la escuela. El reclamo a la presente estrategia, aunque es para confrontar una contingencia, se pregunta ¿qué tipo de estudiante se espera favorecer?; ¿por qué las estrategias por defecto favorecen siempre el estudiante ideal que aspira la escolarización? o ¿por qué no pensar una estrategia desde el lugar de partida desde el cual inicia la carrera un niño o una niña en situación de discapacidad?

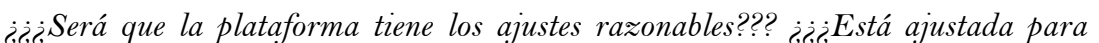

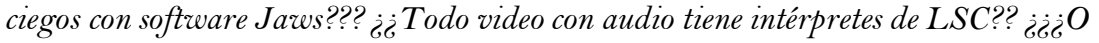

$$
\begin{aligned}
& \text { están subtitulados??? ¿̇ंढे } \Upsilon \text { algo no menos importante, será que habrá un plan de }
\end{aligned}
$$

\subsection{Exhortaciones}

\subsubsection{Mayor inversión del Estado, pero con enfoque territorial}

La política educativa digital debe permitir acceso libre a Internet con fines educativos para el uso de niños y niñas en sus hogares y en las escuelas. El costo de Internet debe ser más asequible para las personas desfavorecidas. Para el sector rural parece haber una educación desfinanciada que no permite impulsar proyectos de infraestructura ni conectividad tecnológica. Las políticas deben considerar enfoques diferenciales y territoriales en los cuales apelar a la equidad es habilitar, de cantidad y calidad, a todos para lograr las mismas oportunidades de aprender. La crisis no debe ser disimulada con la expresión "no estábamos preparados para esto", por el contrario, la crisis deja en evidencia la escasa ejecución de las promesas políticas. El talento humano docente dispuesto en las escuelas de todo el territorio se activa en la medida que se garanticen las condiciones para ejercer su labor.

Red de internet abierta que de acceso únicamente a los computadores y tabletas registradas por el gobierno nacional para colegios. Así los niños mas pobres podrán sus padres ir al colegio o una persona encargada podrá llevar las tabletas y los computadores portátiles. A las casas de estos estudiantes. Con el fin de poder tomar las clases virtuales. Computadores para educar deben tener toda la información de los email de los computadores para así dar acceso al internet gratuito. (1:65, \#22marzo)

\subsubsection{Buscar equidad}

La estrategia favorece a los sectores con mayores comodidades y deja en suspenso a aquellos que, por el contrario, no cuentan con las facilidades. Asegurar plataformas digitales es asegurar tan solo una porción del país, pero invisibiliza otra porción. La estrategia evidencia una profunda desigualdad reproducida con cada acción ministerial que desconoce las necesidades aún no satisfechas de muchos sectores de la sociedad. La competitividad debe basarse en mayor equidad, brindar la oportunidad a todos. La estrategia digital presenta un problema en la concepción misma porque hay un problema: no beneficia a todos. Entonces, algo anda mal en la planeación de la política educativa. Con esto naturalizamos la desigualdad como algo irremediable y no como producto de una 
construcción basada en las intenciones de favorecer unos pocos en desmedro de muchos otros.

\begin{abstract}
Sin Internet y sin acceso a elementos para implementación en las comunidades vulnerables, es educación para las clases más acomodadas económicamente, no se puede pretender educar con estas tecnologías si la mayoría de los estudiantes no tienen el acceso, eso es excluyente. (3:12, \#2 1abril)
\end{abstract}

Las propuestas son pensadas para un estudiante ideal que corresponde a un solo tipo o modelo de escolarización. Desconoce otras condiciones de escolarización tan diversos como cantidad de regiones y poblaciones habitan en Colombia.

El ministerio no conoce de las cuatro paredes de su oficina. Para afuera la realidad
del país es otra totalmente distinta a lo que ellos "conocen" pero preparémonos como
siempre con nuestras uñas haciendo lo mejor posible por nuestros estudiantes. En fin,
que nadie más se afana por ellos... (2:45, \#31marzo)

\title{
4.3.3 Compromiso intersectorial
}

El llamado es a las empresas de telecomunicaciones para que demuestren mayor compromiso con el trabajo articulado por la educación. El gravamen de impuestos debe eliminarse para la intención de comprar artículos para la conectividad de los hogares más desfavorecidos, esto hace más asequible el acceso digital. La conectividad digital para hogares no debe pensarse como un nicho comercial, sino parte de un lugar estratégico para apostar por mayor equidad a través del control de los costos que ofrecen las compañías de telecomunicaciones.

¿Cuándo las multinacionales@ClaroColombia@Tigo_Colombia@MovistarCo
van a aportarsugranito de arena, comolohizo@ETBqueamplio el ancho de banda
a los que tienen menos de 100mb para que los niños y niñas se puedan conectary
trabajar en mejores condiciones? (1:46, \#18marzo)

4.3.4. Asegurar las necesidades básicas insatisfechas

El ciudadano exhorta las necesidades de transporte, salud, servicios básicos del hogar aún no asegurados. Es paradójico y cómico ver que hay una versión lejana de país que marcha a un ritmo como reza el eslogan presidencial "El Futuro es de todos", pero otra está paquidérmica detenida en el pasado. El llamado ciudadano es a razonar, a no vivir en un país de fantasías de lo que debería ser, con facilidad asequible y accesible para pocos, pero negado e imposible para muchos otros. Para sectores sociales desfavorecidos y olvidados escuchar una estrategia digital para confrontar el cierre de escuelas es confrontarlos con un sinsentido y con la verdadera necesidad y urgencia existencial para ellos: suplir las necesidades básicas para sobrevivir. La paradoja digital como apuesta para enfrentar el futuro y propiciar la calidad de la educación se confronta con políticas compensatorias (alimentación escolar, planta docente o infraestructura) que en otrora prometieron calidad, pero que aún hoy no consiguen ser universales. ¿Qué hace pensar que la moda digital alcance la calidad de la educación no lograda?; ¿qué hace pensar que, a diferencia de las políticas compensatorias, que en otrora prometieron universlidad, la política educativa digital si alcanzará a ser universal? Una asincronía entre las promesas del pasado no cumplidas y las promesas de futuro.

¿Qué graciosos!, y ¿̇cómo se atenderán las comunidades que escasamente tiene fluido eléctrico? La economía naranja de Duque habla de prepararnos para la tecnología $5 G$ y en los campos colombianos buscamos la montaña más alta para lograr la EDGE. No hay garantías fisicas (baños, infraestructura), ni garantías en la nutrición (PAE) y iasí pretenden apoyar la educación! (1:67, \#22marzo) 


\section{Discusión}

Las réplicas ciudadanas categorizadas robustecen la evidencia en torno al agravamiento de las desigualdades educativas en época de covid-19. La presente investigación ratifica las conclusiones de Garrido (2020) y Quiroz (2020) que hacen referencia a las desigualdades precedentes, ahora exacerbadas. Evidenciamos que la deuda histórica del Estado colombiano con las periferias, la ruralidad y los sectores urbanos desfavorecidos está más vigente que nunca. Por tanto, los diagnósticos continuados de desigualdades abordados por las ciencias sociales latinoamericanas antes que literatura panfletaria, como lo hacen ver los sectores políticos conservadores y las derechas más radicales, son altavoz de realidades precarias, con esperanzas, pero constreñidas por voluntades políticas que no cuajan en el verdadero propósito de la equidad social.

Agreguemos a lo anterior, que el presente estudio remarcó en una fracción de esta indeseable realidad latinoamericana. En particular, la política educativa digital tan connotada y publicitada, pero inaccesible para los sectores sociales de siempre que ven negados o, en el mejor de los casos, ralentizados los beneficios educativos de los que goza la población más favorecida. Las réplicas ciudadanas dan soporte empírico a las conclusiones ofrecidas en la investigación documental de Sánchez-Gómez y colaboradores (2020). En efecto, el estado de cuarentena y el cierre masivo de escuelas significaron apelar a la propuesta digital como solución, por defecto, mayor dificultad para sectores sociales encarnados en la figura del niño y la niña de los suburbios, la familia rural o el docente que sirve a las comunidades indígenas. En acuerdo con Cáceres-Muñoz, JiménezHernández y Martín-Sánchez (2020) y Álvarez y otros (2020), aprendimos que la rápida salida tecnológica a los problemas (im)predecibles de la educación no deja algo más que un haz de incertidumbres, voces negadas y posibilidades no encontradas.

Advertimos con esta investigación la homogeneidad de la política educativa digital. Las réplicas ciudadanas son una misma voz que, al unísono, reclaman focalización, personalización y enfoque territorial para la justificada transformación digital (OCDE, 2019), no solo aquella de emergencia en ocasión al covid-19, sino la pretendida en las actuales políticas económicas en Colombia (Departamento Nacional de Planeación de Colombia, 2018, 2019). Las voces de ciudadanos y ciudadanas en redes sociales resisten la visión unívoca del aprendiz digital (Williamson, 2018), bien dotado de recursos, auto motivado, con gran apertura para experimentar en la incertidumbre, pero con escasa consideración de las innegables diferencias socioeconómicas y culturales de origen que hacen reprochable la universalización del aprendiz (Selwyn, 2016).

Al respecto, con los estudios de Mérida y Acuña (2020) y Gallegos y Tinajero (2020), o en las conclusiones de Rodríguez (2020), no solo hallamos simpatías en torno a la reflexión por la no homogeneización, sino que además evocamos las necesarias políticas de subjetividad: para una educación latinoamericana justa la política educativa no deberá advertir a la ciudadanía como una masa homogénea que deviene en las mismas necesidades, sino un reto que "deberá trabajar sobre las representaciones que los actores tienen acerca de las principales categorías vinculadas a [su] trayectoria educativa" (Tedesco, 2012, pp. 210-211).

Trabajar sobre las representaciones de los actores implica devolver confianza, capacidad para narrar y elaborar proyectos. En el respectivo orden, la figura del Estado, el intelectual de las ciencias sociales y de la escuela son irremplazables. 
Respecto del primero, el presente estudio nos permitió develar réplicas ciudadanas que exhortan por la confianza a un Estado deslucido por la corrupción, la burocracia y el clientelismo con el que se gestionan los bienes públicos. El llamado es unívoco para visibilizar otras ciudadanías que merecen reconocimiento en la vida pública, ricas en experiencias y expectativas, pero con carencias que valen la pena atenderse. Segundo, aprendimos a valorar la capacidad de narración inherente a la ciudadanía. Narraciones que, de ser capitalizadas, constituyen el germen para la producción teórica de las ciencias sociales. Una producción cuyo interés será el autoconocimiento como posibilidad emancipadora (Santos, 2009), solo de la mano de intelectuales de retaguardia: "atentos a las necesidades y aspiraciones de los ciudadanos comunes y teorizar a partir de ellas. De lo contrario, los ciudadanos estarán indefensos" (Santos, 2020, p. 41). Por último, hallamos un reclamo por una escuela renovada que, en pospandemia, tome nota del problema de su rediseño en perspectiva de proyectos que promueven aprendizajes hacia una sociedad digitalizada y del siglo XXI (Maggio, 2018; Pinto, 2019; Rivas, 2017, 2019). Las réplicas ciudadanas, más allá que la garantía en infraestructura y el acceso digital, conjuran el cambio escolar, el desarrollo de una cultura digital, la formación docente y la relación escuela-comunidad como eslabones que fortalecen la adopción contextual de la política digital y amplían las fronteras de la educación más allá de las murallas del edificio escolar (Fernández-Enguita, 2018).

En esta perspectiva resaltamos la continuidad empírica que representa nuestro estudio con las conclusiones de la investigación documental de Lugo y otros (2020). Coincidimos con una necesaria plataforma educativa digital que diversifique los tradicionales escenarios de la escuela. Además, en la escalabilidad y gradualidad, la conectividad universal, la asequibilidad digital y el rediseño curricular como condiciones ineludibles para una política educativa digital puesta a prueba en pandemia y con lecciones aprendidas de cara a la pospandemia.

\section{Conclusiones}

Primero, el presente estudio prometió categorizar las réplicas ciudadanas con el ánimo de revelar las desigualdades educativas agravadas por el cierre masivo de las escuelas y por la aplicación de una estrategia digital homogénea para confrontar la excepcional situación. Valoramos de este primer objetivo la riqueza de contenidos expuestos en las réplicas ciudadanas de Twitter y Facebook. Hallamos un espacio fértil de necesaria incursión en las investigaciones en ciencias sociales que implica ampliar el abanico de potenciales fuentes de información. De hacerlo tendremos una perspectiva más amplia de la realidad social. Limitarnos a las fuentes tradicionales de información, de lo contrario, implicará la pérdida de voces ciudadanas que valen la pena capitalizar por el alto valor informativo que traen consigo, como lo apreciáramos en el apartado de resultados.

Segundo, el estudio prometió discutir, en el marco de las réplicas, las expresiones de desigualdad educativa. Al final, creemos satisfecha la tarea en la medida que aportó al propósito del presente número extraordinario de la Revista Internacional de Educación para la Justicia Social: "las consecuencias del cierre de escuelas por el covid-19 en las desigualdades educativas". Ofrecimos evidencia para robustecer la denuncia que estima a los sectores sociales históricamente desfavorecidos como los mismos que padecen con más intensidad las consecuencias de la actual excepcionalidad. Además, reunimos referencias para atestiguar en contra de las políticas educativas homogéneas, en particular la digital, 
que enmarcan los fines de la escuela en una figura unívoca del aprendiz que niega otras posibilidades tan diversas y alejadas como disímiles en Latinoamérica.

Tercero, ¿qué lección aprendida surge de las réplicas ciudadanas a la estrategia \#AprenderDigital para una escuela pospandemia? La respuesta es otra pregunta con un amago de propuesta que habrá que cultivar: ¿Qué escuela esperamos construir cuando las puertas de ésta una vez más estén abiertas a millones de niños y niñas en nuestras latitudes latinoamericanas, en especial, las que corresponden al territorio colombiano? Una escuela que no acepte la solución tecnológica como salida fácil a todas sus críticas, sino que avance hacia la construcción colectiva de voluntades para habilitar con tecnología condiciones individuales e institucionales para unos mejores aprendizajes. Implica avanzar hacia la educabilidad digital como una noción para generar proyectos de formación que visibilicen las propias necesidades, habiliten las oportunidades y propicien la esperanza entre tantas desigualdades. Al respecto, la docencia tiene una responsabilidad única e histórica de superar la veda del solucionismo tecnológico. La accesibilidad digital cobra sentido en el mismo momento que la pregunta: “icon qué educar?”, es complementada con "la actitud, la reflexión y el saber pedagógico para no solo ver el árbol, sino percibir el bosque” (Suárez, 2019, párr.1). Para este fin, la elaboración de proyectos escolares con tecnologías digitales que vinculan la gestión y la planificación, el desarrollo profesional de los docentes, el desarrollo curricular, la cultura digital y la relación escuela-comunidad posibilitan que, aún en condiciones adversas, los aprendizajes escolares sean posibles (Lugo y Kelly, 2011; Tedesco, 2012).

Cuarto, la investigación ratifica el uso humanitario (UNESCO, 2020b), que significan las tecnologías digitales en el marco de la actual emergencia sanitaria para otorgar espacio de expresión, reivindicar voces desconocidas; para que el ciudadano no vea resignada su posibilidad de denunciar y exhortar a los gobiernos. Por último, para apreciar la tecnología digital más allá de la dicotomía uso/mal uso y visionarlo como una necesaria oportunidad para el goce de los derechos, en particular el derecho de los más jóvenes en Internet (Pavez, 2014).

Terminamos inspirados en De Sousa Santos (2020), ¿la verdad de las políticas educativas digitales se conoce mejor en situaciones normales o en situaciones excepcionales? Con el mismo autor respondemos: ambas situaciones inducen conocimiento, sin embargo, iccuán dispuestos estaremos en unos años para recordar estos tiempos de excepcionalidad que evidenciaron las desigualdades que parecen paisaje en tiempos de "normalidad"?!

\section{Referencias}

Álvarez, M., Gardyn, N., Iardelevsky, A. y Rebello, G. (2020). Segregación educativa en tiempos de pandemia: Balance de las acciones iniciales durante el aislamiento social por el Covid-19 en Argentina. Revista Internacional de Educación para la Justicia Social, 9(3), 25-43. https://doi.org/10.15366/riejs2020.9.3.002

Baptista, L., Loeza, C., Almazán, A., López, V. y Cárdenas, J. (2020). Encuesta nacional a docentes ante el Covid-19. Retos para la educación a distancia. RLEE Nueva Época, 5O(e), 41-88.

Cáceres-Muñoz, J., Jiménez-Hernández, A. S. y Martín-Sánchez, M. (2020). Cierre de escuelas y desigualdad socioeducativa en tiempos del Covid-19. una investigación exploratoria en clave internacional. Revista Internacional de Educación para la Justicia Social, 9(3), 199-221. https://doi.org/10.15366/riejs2020.9.3.011 
Cervantes, E. y Gutiérrez, P. (2020). Resistir la Covid-19. Intersecciones en la educación de Ciudad Juárez, México. Revista Internacional de Educación para la Justicia Social, 9(3), 7-23. https://doi.org/10.15366/riejs2020.9.3.001

Charmaz, K. (2006). Constructing grounded theory. A practical guide through qualitative analysis. Sage.

Departamento Administrativo Nacional de Estadística. (2018). Indicadores básicos de TIC en hogares. Departamento Administrativo Nacional de Estadística.

Departamento Nacional de Planeación de Colombia. (2019). Política nacional para la transformación digital e inteligencia artificial. Departamento Nacional de Planeación de Colombia.

Dirección Nacional de Planeación de Colombia. (2020). Tecnologías para aprender: Política nacional para impulsar la innovación en las prácticas educativas a través de las tecnologías digitales. Departamento Nacional de Planeación de Colombia.

Fernández-Enguita, M. (2018). Más escuela y menos aula. La innovación en la perspectiva de un cambio de época. Morata.

Freeman, M. (2017). Modes of thinking for qualitative data analysis. Routledge. https://doi.org/10.4324/9781315516851

Friese, S. (2019). Qualitative data analysis with Atlas ti. Sage.

Gallegos, I. y Tinajero, M. (2020). Resiliencia y demandas de política educativa durante la contingencia sanitaria. Revista Latinoamericana de Estudios Educativos, 5O(e), 121-142.

Garrido, M. D. (2020). Educar en tiempos de pandemia: Acentuación de las desigualdades en el sistema educativo chileno. Revista Caminhos da Educação: Diálogos, Culturas e Diversidades, 2(2), 43-68. https://doi.org/10.26694/caedu.v3i1

Krüger, N. (2019). La segregación por nivel socioeconómico como dimensión de la exclusión educativa: 15 años de evolución en América Latina. Archivos Analíticos de Políticas Educativas, $27(8)$. https://doi.org/10.14507/epaa.27.3577

Lugo, M. T., Ithurburu, V., Sonsino, A. y Loiacono, F. (2020). Políticas digitales en educación en tiempos de Pandemia: Desigualdades y oportunidades para América Latina. Revista Electrónica de Tecnología Educativa, 73, 23-36. https://doi.org/10.21556/edutec.2020.73.1719

Lugo, M. T. y Kelly, V. (2011). La matriz TIC. Una herramienta para planificar las tecnologías de la información y la comunicación en las instituciones educativas. UNESCO.

Maggio, M. (2018). Habilidades del siglo XXI: Cuando el futuro es hoy. Fundación Santillana.

Mérida, Y., y Acuña, L. (2020). Covid-19, pobreza y educación en Chiapas: Análisis a los programas educativos emergentes. Revista Internacional de Educación para la Justicia Social, 9(3), 61-82. https://doi.org/10.15366/riejs2020.9.3.004

Ministerio de Educación de Colombia. (2020). Estrategia aprender digital. Ministerio de Educación de Colombia.

Murillo, F. J. y Martínez-Garrido, C. (2017). Segregación social en las escuelas públicas y privadas en América Latina. Educação E̊ Sociedade, 38(140), 727-750. https://doi.org/10.1590/ESO101-73302017167714

Murillo, F. J. y Martínez-Garrido, C. (2018). Evolución de la segregación socioeconómica de las escuelas de América Latina. Estudios pedagógicos, 44(1), 157-179. https://doi.org/10.4067/So718-07052018000100157

OCDE. (2019). Vectors of digital transformation. OECD Publishing. 
Pavez, M. I. (2014). Los derechos de la infancia en la era de internet: América Latina y las nuevas tecnologías. CEPAL.

Pinto, L. (2019). Rediseñar la escuela para y con las habilidades del siglo XXI. Fundación Santillana.

Quiroz, C. (2020). Pandemia Covid-19 e inequidad territorial: El agravamiento de las desigualdades educativas en Chile. Revista Internacional de Educación para la Justicia Social, 9(3).

Ramos-Huenteo, V., García-Vásquez, H., Olea-González, C., Lobos-Peña, K. y Sáez-Delgado, F. (2020). Percepción docente respecto al trabajo pedagógico durante la Covid-19. CienciAmérica, 9(2), 334-353. https://doi.org/10.33210/ca.v9i2.325

Rivas, A. (2015). América Latina después de PISA: Lecciones aprendidas de la educación en siete países 2000-2015. CIPPEC-Natura-Instituto Natura.

Rivas, A. (2017). Cambio e innovación educativa: Las cuestiones cruciales. Fundación Santillana.

Rivas, A. (2019). ¿Qué hay que aprender hoy? De la escuela de las respuestas a la escuela de las preguntas. Fundación Santillana.

Rodríguez, P. (2020). ¿Políticas uniformes y universales de acceso a la tecnología revierten desigualdades educativas? Revista Internacional de Educación para la Justicia Social, 9(3).

Sánchez-Gómez, J., Quiroga, K. y Ospina, P. (2020). Desafíos tecnológicos para el sector educativo en América Latina en tiempos de pandemia. Programa de Investigación de Política Exterior Colombiana.

Sánchez, M., Martínez, A., Torres, R., de Agüero, M., Hernández, A., Benavides, M., .. . y Jaimes, C. (2020). Retos educativos durante la pandemia de COVID-19: Una encuesta a profesores de la UNAM. Revista Digital Universitaria, 21(3), 1-24.

Santos, B. D. S. (2009). Una epistemología del sur. La reinvención del conocimiento y la emancipación social. Siglo XXI Editores.

Santos, B. D. S. (2020). La cruel pedagogía del virus. Consejo Latinoamericano de Ciencias Sociales.

Selwyn, N. (2016). Is technology good for education? Polity.

Suárez, C. (2019). El árbol y el bosque. En torno a la pedagogía digital. https://bit.ly/384hDHY

Taylor, S. y Bodgan, R. (1987). Introducción a los métodos cualitativos de investigación. La búsqueda de significados. Paidós Ibérica.

Tedesco, J. C. (2012). Educación y justicia social en América Latina. Fondo de Cultura Económica.

UNESCO. (2020a). Interrupción educativa y respuesta al COVID-19. UNESCO.

UNESCO. (2020b). La UNESCO reafirma la necesidad de la universalidad de Internet, en medio de la intensificación de las amenazas. UNESCO.

Williamson, B. (2018). Big data en educación. El futuro digital del aprendizaje, la política y la práctica. Morata. 


\section{Breve CV del autor}

\section{Edwar Camilo Lopera-Zuluaga}

Investigador y profesor de escuela primaria pública en la Institución Educativa Liceo Antioqueño del municipio de Bello (Colombia). Magíster en Educación y Desarrollo Humano de la Universidad de Manizales-Cinde (Sabaneta, Colombia). Doctorando en Ciencias Sociales de la Universidad Pontificia Bolivariana (Medellín, Colombia). Su trayectoria educativa se centra en proyectos comunitarios para la formación ciudadana, el ejercicio docente activo en escuelas públicas y la militancia en el sindicato de trabajadores de la educación de Antioquia-Adida-. Sus actuales intereses investigativos se basan en la fundamentación de la noción de Educabilidad digital para intervenir en la formulación de proyectos educativos de formación digital de niños y niñas en condición de segregación escolar socioeconómica. ORCID ID: https://orcid.org/0000-0003-3316-6544. Email: edwar.lopera@upb.edu.co 